\title{
Aortic valve replacement for a case of anomalous origin of the left coronary artery from posterior sinus of Valsalva with intramural aortic course
}

Naoto Morimoto, MD, Yutaka Okita, MD, Kenji Okada, MD, Teruo Yamashita, MD, and Masamichi Matsumori, MD, Kobe, Japan

W

e present a case of aortic valve replacement with a rare combination of the anomalous left coronary artery (LCA) and the intramural coronary artery.

\section{Clinical Summary}

A 72-year-old man had been intermittently febrile for 6 months. After admission, transthoracic echocardiography revealed a vegetation attached to the noncoronary cusp, with moderate aortic insufficiency. Transesophageal echocardiography demonstrated that the left main trunk (LMT) had an aortic intramural course shortly from its origin to the midportion of left sinus and then distributed normally to the left ventricle (Figure 1). Alpha-hemolytic Streptococcus species was isolated from a series of blood cultures. Despite antibiotic therapy for 3 weeks, the vegetation continued growing, and surgical intervention was determined.

The operation was performed with moderate hypothermic cardiopulmonary bypass. After chemical cardiac arrest, the aorta was incised transversely $2 \mathrm{~cm}$ above the sinotubular junction. All three leaflets were perforated and excised. The LCA was found to be arising in the posterior sinus of Valsalva close to the nonleft commissure, and the LMT was embedded in the aortic wall. The right coronary artery ostium deviated slightly anteriorly from its normal anatomic position. The infected aortic valve was replaced by a subcoronary technique with a $23-\mathrm{mm}$ Freestyle aortic root bioprosthesis (Medtronic, Inc, Minneapolis, Minn). The proximal suture line was placed on the subaortic horizontal plain with interrupted sutures. Each commissural post of the bioprosthesis was secured carefully to avoid obstruction of the anomalous coronary ostia by rotating $60^{\circ}$ counterclockwise. The post near the right coronary orifice was slightly distorted to keep some space from the orifice (Figure 2,a). The distal suture line was placed with running sutures, starting at the nadir of the left sinus and working up each side toward the commissures.

From the Division of Cardiovascular, Thoracic, and Pediatric Surgery, Kobe University Graduate School of Medicine, Kobe, Japan.

Received for publication July 3, 2005; accepted for publication Aug 3, 2005.

Address for reprints: Yutaka Okita, MD, Division of Cardiovascular, Thoracic, and Pediatric Surgery, Kobe University Graduate School of Medicine, 7-5-2 Kusunoki-cho, Chuo-ku, Kobe 650-0017, Japan (E-mail: yokita@med.kobe-u.ac.jp).

J Thorac Cardiovasc Surg 2005;130:1713-4

$0022-5223 / \$ 30.00$

Copyright (C) 2005 by The American Association for Thoracic Surgery

doi:10.1016/j.jtcvs.2005.08.003

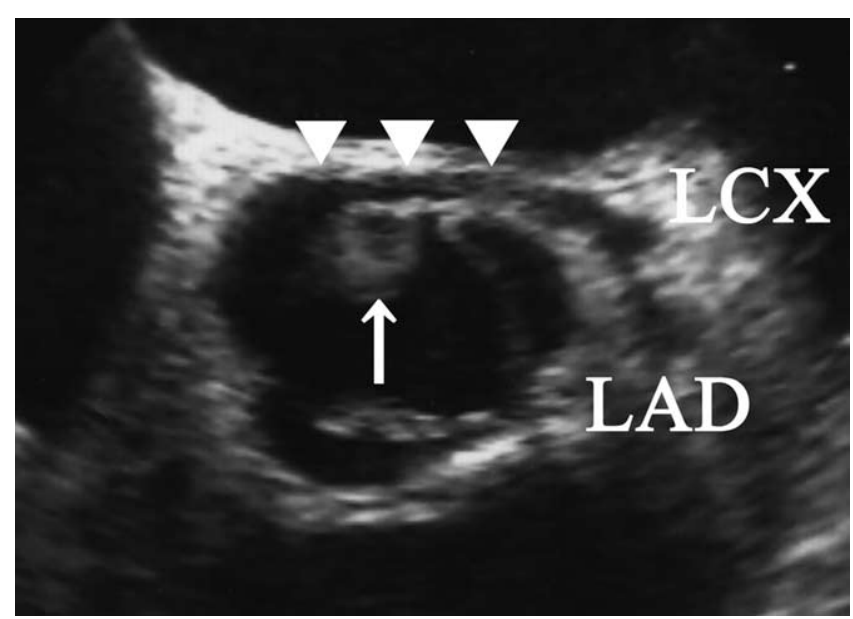

Figure 1. Preoperative transesophageal echocardiography showing anomalous origin of the left coronary artery with an intramural course (triangle) and the vegetation on the ventricular side of the aortic valve (arrow). LAD, Left anterior descending artery; LCX, left circumflex artery.

After weaning from cardiopulmonary bypass, the hemodynamics suddenly deteriorated, and transesophageal echocardiography showed hypokinesis of the anteroseptal wall, suggesting the damage to the LCA. Cardiopulmonary bypass was reestablished, and the aorta was opened again. The smooth passage of a $6-\mathrm{mm}$ selective coronary perfusion catheter into LCA showed no mechanical obstruction in this condition. Once the heart was beating and aortic root pressure was increased, however, the intramural segment of the LMT seemed to be stretched by the bioprosthesis, resulting in luminal narrowing. The stentless valve was excised, and a 21-mm CarboMedics Top Hat mechanical prosthesis (CarboMedics Inc, Austin, Tex) was implanted in the subannular position with interrupted sutures. The patient was weaned from cardiopulmonary bypass without any incidents. The postoperative course was uneventful. After 3 weeks of antimicrobial therapy, the patient was discharged. He remains alive at 3 years without any sign of recurrence of either infection or myocardial ischemia.

\section{Discussion}

Anomalous coronary arteries are usually an incidental finding during conventional coronary angiography. An aberrant LCA from the posterior sinus is extremely rare and has not been previously reported. In our case, the LMT also had an aortic intramural course 
a)

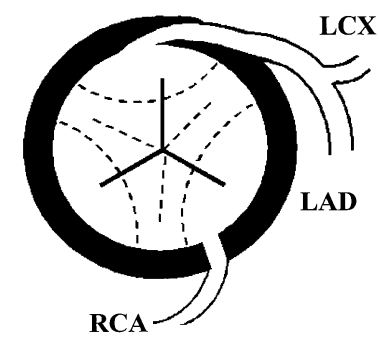

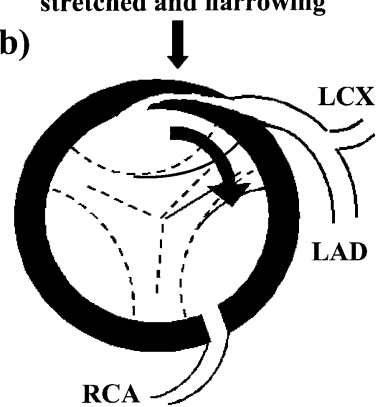

Figure 2. a, Implantation technique. By rotation of bioprosthesis (perforated line), anomalous LCA arising from near nonleft commissure was left intact. Commissural post near right coronary orifice was slightly distorted to keep some space from orifice. $b$, Mechanism of LCA malperfusion. During beating, distorted commissural posts of bioprosthesis were forced to displace symmetrically. Intramural segment of anomalous LCA was stretched and narrowed by rotation of post near origin of LCA. RCA, Right coronary artery; $L A D$, left anterior descending coronary artery; LCX, left circumflex artery.

from its origin to the midpoint of the left sinus of Valsalva. In our review of the literature, we found 11 cases of aortic valve replacement with anomalous coronary artery origin. With respect to the selection of the prosthesis, a mechanical valve was implanted in 6 cases, ${ }^{1-4}$ a stented bioprosthesis in 3 cases, ${ }^{5}$ and a stentless bioprosthesis in 2 cases (full root technique in 1 and subcoronary in 1). ${ }^{5}$ In 6 of 8 reported cases with a mechanical valve or stented bioprosthesis, the anomalous origin of the LCA was intraoperatively or postoperatively compressed by the rigid prosthetic ring, which caused myocardial ischemia and sudden death. All these valves were replaced in the intra-annular position with the prosthesis,

which fit the diameter of the aortic annulus as measured with the sizer. Adjustments in other successful cases included additional coronary artery bypass grafting, subannular positioning, and intraannular positioning with a prosthesis smaller by one size than measured. At first we performed subcoronary implantation of stentless bioprosthesis to avoid coronary artery compression by the rigid prosthetic ring. The distal suture line was placed carefully to avoid interfering with the intramural LMT. However, LCA blood flow was adversely affected, although it was not obstructed by the suture line. We believe the mechanism is as follows: The rotation and deformation of the bioprosthesis to avoid the obstruction of coronary ostia made the aortic sinus wall of intramural segment stretched after beating, which resulted in luminal narrowing of the intramural segment (Figure 2, b).

This case highlights special surgical considerations when aortic valve replacement is performed on the patient with coronary artery anomaly, including selection of the type of prosthesis, implantation techniques, and possible selection of a smaller prosthetic ring. When coronary artery ischemia is suspected, coronary artery bypass grafting or reoperative replacement of the prosthesis should be considered.

\section{References}

1. Roberts WC, Morrow AG. Compression of anomalous left circumflex arteries by prosthetic valve fixation rings. $J$ Thorac Cardiovasc Surg. 1969;57:834-8

2. Flores RM, Byrne JG. Aortic valve replacement with an anomalous left circumflex coronary artery encircling the aortic annulus. $J$ Thorac Cardiovasc Surg. 2001;12:1396-7.

3. Marchena EJ, Russo CD, Wozniak PM. Compression of an anomalous left circumflex artery by a bioprosthetic valve ring. J Cardiovasc Surg. 1990;31:52-4.

4. Misawa Y, Saito T, Oki S. Management of anomalous right coronary arteries encountered during aortic valve surgery. Eur J Cardiothorac Surg. 2002;21:102-4.

5. O'Blenes SB, Feindel CM. Aortic root replacement with anomalous origin of the coronary arteries. Ann Thorac Surg. 2002;73:647-9. 